\title{
BS methods: a new class of spline collocation BVMs
}

\author{
Francesca Mazzia, Alessandra Sestini, Donato Trigiante
}

\begin{abstract}
BS methods are a recently introduced class of Boundary Value Methods which is based on B-splines and can also be interpreted as spline collocation methods. For uniform meshes the coefficients defining the $k$-step BS method are just the values of the $(k+1)$-degree uniform B-spline and B-spline derivative at its integer active knots; for general nonuniform meshes they are computed by solving local linear systems whose dimension depends on $k$. An important specific characteristic of the BS methods is the possibility to associate a spline of degree $k+1$ and smoothness $C^{k}$ to the numerical solution produced by the $k$-step method of this class. Such spline collocates the differential equation at the knots, shares the convergence order with the numerical solution and can be computed with negligible additional computational cost.

In this talk a survey on such methods will be given, by presenting the general definition, the convergence and stability features, and by introducing the strategy for the computation of the coefficients in the B-spline basis defining the associated spline. Finally, some related numerical results will be also presented.
\end{abstract}

\section{References}

F. Mazzia, A. Sestini and D. Trigiante, "B-spline Linear Multistep Methods and their Continuous Extensions", Siam J. of Numerical Analysis 44, No. 5, 1954-1973, (2006).

F. Mazzia, A. Sestini and D. Trigiante, "BS Linear Multistep Methods on Non-uniform Meshes", JNAIAM 1, 131-144, (2006).

F. Mazzia, A. Sestini and D. Trigiante," The continuous extension of the BS linear multistep methods on non-uniform meshes ", in press in APNUM (2008),

doi:10.1016/j.apnum.2008.03.036. 